\title{
Learning Alters the Tuning of Functional Magnetic Resonance Imaging Patterns for Visual Forms
}

\author{
Jiaxiang Zhang, ${ }^{1,2}$ Alan Meeson, ${ }^{1}$ Andrew E. Welchman, ${ }^{1}$ and Zoe Kourtzi ${ }^{1}$ \\ ${ }^{1}$ School of Psychology, University of Birmingham, Edgbaston, Birmingham B15 2TT, United Kingdom, and ${ }^{2}$ Medical Research Council, Cognition and Brain \\ Sciences Unit, Cambridge CB2 7EF, United Kingdom
}

Learning is thought to facilitate the recognition of objects by optimizing the tuning of visual neurons to behaviorally relevant features. However, the learning mechanisms that shape neural selectivity for visual forms in the human brain remain essentially unknown. Here, we combine behavioral and functional magnetic resonance imaging ( $\mathrm{fMRI}$ ) measurements to test the mechanisms that mediate enhanced behavioral sensitivity in the discrimination of visual forms after training. In particular, we used high-resolution fMRI and multivoxel pattern classification methods to investigate fine learning-dependent changes in neural preference for global forms. We measured the observers' choices when discriminating between concentric and radial patterns presented in noise before and after training. Similarly, we measured the choices of a pattern classifier when predicting each stimulus from fMRI activity. Comparing the performance of human observers and classifiers demonstrated that learning alters the observers' sensitivity to visual forms and the tuning of fMRI activation patterns in visual areas selective for task-relevant features. In particular, training on low-signal stimuli enhanced the amplitude but reduced the width of pattern-based tuning functions in higher dorsal and ventral visual areas. Thus, our findings suggest that learning of visual patterns is implemented by enhancing the response to the preferred stimulus category and reducing the response to nonpreferred stimuli in higher extrastriate visual cortex.

\section{Introduction}

Detecting and identifying meaningful objects in clutter is a critical skill for interactions and survival in complex environments. Although these processes appear fast and effortless, the computational challenges of visual recognition are far from trivial. For example, the recognition of coherent objects entails segmentation of relevant features from clutter and discrimination of highly similar features belonging to different objects. Learning has been suggested to facilitate these processes by tuning neural selectivity to behaviorally relevant visual features (for review, see Gilbert et al., 2001; Kourtzi and DiCarlo, 2006). However, the learning mechanisms that shape neural selectivity for visual forms in the human brain remain essentially unknown. In particular, learning-dependent changes in neural selectivity (i.e., sharpening of neuronal tuning to a visual stimulus) may result from three different possible mechanisms: enhanced response to the preferred stimulus, decreased response to the nonpreferred stimulus, or a combination of the two. Here, we combine behavioral and fMRI measurements to test which of these neural mechanisms mediate learning of forms in the human visual cortex. We exploit the sensitivity of high-resolution fMRI and multivoxel

Received April 29, 2010; revised Aug. 17, 2010; accepted Aug. 19, 2010.

The research leading to these results has received funding from Biotechnology and Biological Sciences Research Council Grants BB/D52199X/1 and BB/E027436/1 and European Community Seventh Framework Programme Grant FP7/2007-2013 under Grant Agreement 214728. Thanks to M. Dexter for help with the development of analysis tools.

Correspondence should be addressed to Zoe Kourtzi, University of Birmingham, School of Psychology, Edgbaston, Birmingham B15 2TT, UK. E-mail: z.kourtz@@ham.ac.uk.

DOI:10.1523/JNEUROSCI.2204-10.2010

Copyright $\odot 2010$ the authors $\quad 0270-6474 / 10 / 3014127-07 \$ 15.00 / 0$ pattern classification analysis (MVPA) methods to investigate fine learning-dependent changes in neural preference at the level of large neural populations in the human visual cortex as revealed by fMRI.

In particular, we used a morphing stimulus space that is generated by varying the spiral angle between radial and concentric patterns resulting in stimuli that vary in their similarity (see Fig. 1A). Observers were presented with stimuli at different amounts of background noise (i.e., high-signal vs lowsignal stimuli) and judged whether they resembled a concentric or radial visual pattern. We measured the observers' choices (i.e., proportion concentric) with high-signal stimuli and compared them with those with low-signal stimuli before and after training. Similarly, we measured the choices of a pattern classifier, that is, the proportion of patterns on which the classifier predicted each stimulus from fMRI activity pooled across voxels. We investigated the link between behavioral and fMRI learning changes by comparing psychometric functions and fMRI pattern-based tuning functions before and after training.

Our findings demonstrate that training alters the observers' sensitivity to visual forms and fMRI selectivity in higher dorsal and ventral visual areas. Specifically, training on lowsignal stimuli enhanced the amplitude but decreased the width of pattern-based tuning in higher dorsal and ventral visual areas. These findings suggest that learning enhances behavioral sensitivity to visual forms and fMRI sensitivity in higher visual areas by enhancing neural responses to behaviorally relevant features, whereas decreasing responses to nonpreferred features. 


\section{Materials and Methods}

Participants

Ten observers participated in the study (two males, eight females; age range, 19-37 years). All observers had normal or corrected-tonormal vision, gave written informed consent, and were paid for their participation. The data from one observer were excluded as a result of low behavioral performance after training and from a second observer as a result of poor fMRI signals even for high-signal stimuli. The study was approved by the local ethics committee.

\section{Stimuli}

Stimuli were Glass patterns (Glass, 1969) generated using previously described methods ( $\mathrm{Li}$ et al., 2009) ensuring that coherent form patterns are reliably perceived for the stimulus generation parameters we used. In particular, stimuli were defined by white dot pairs (dipoles) displayed within a square aperture $\left(7.7^{\circ} \times 7.7^{\circ}\right)$ on a black background $(100 \%$ contrast). The dot density was $3 \%$, and the Glass shift (i.e., the distance between two dots in a dipole) was $16.2 \mathrm{arc}$ min. The size of each dot was $2.3^{\circ} \times 2.3^{\circ}$ arc $\min ^{2}$. For each dot dipole, the spiral angle was defined as the angle between the dot dipole orientation and the radius from the center of the dipole to the center of the stimulus aperture. Each stimulus comprised dot dipoles that were aligned according to the specified spiral angle (signal dipoles) for a given stimulus and noise dipoles for which the spiral angle was randomly selected. The proportion of signal dipoles defined the stimulus signal level. We generated concentric and radial Glass patterns by placing dipoles tangentially (concentric stimuli) or orthogonally (radial stimuli) to the circumference of a circle centered on the fixation dot. Further, we generated intermediate patterns between these two Glass pattern types by parametrically varying the spiral angle of the pattern from $0^{\circ}$ (radial pattern) to $90^{\circ}$ (concentric pattern) (Fig. $1 \mathrm{~A}$ ). Half of the observers were presented with clockwise patterns $\left(0^{\circ}\right.$ to $90^{\circ}$ spiral angle) and half with counterclockwise patterns $\left(0^{\circ}\right.$ to $-90^{\circ}$ spiral angle). A new pattern was generated for each stimulus presented in a trial, resulting in stimuli that were locally jittered in their position.

\section{Design}

All observers participated in three psychophysical training sessions and three fMRI sessions. In the psychophysical sessions, observers were presented with stimuli at $45 \%$ signal level. For the pretraining and posttraining fMRI sessions, observers were presented with stimuli at $45 \%$ signal level (low-signal stimulus sessions), whereas for the third fMRI session, observers were presented with stimuli at $80 \%$ signal level (high-signal stimulus session). The high-signal stimulus session followed the lowsignal stimulus sessions to avoid priming the observers with highly visible versions of the stimuli before training.

\section{Psychophysical training}

Observers were first familiarized with the task during a short practice session (20 trials). Then observers performed one pretest session (one run without feedback), followed by three training sessions that were conducted on different days. Each session comprised five training runs with audio feedback on error trials and was followed by one test run without feedback. Each psychophysical run comprised 160 trials (16 trials per stimulus condition). Stimuli were presented at 10 possible spiral angles $\left(5^{\circ}, 15^{\circ}, 25^{\circ}, 35^{\circ}, 42^{\circ}, 48^{\circ}, 55^{\circ}, 65^{\circ}, 75^{\circ}\right.$, and $\left.85^{\circ}\right)$. Each trial lasted $1.5 \mathrm{~s}$, and the stimulus was presented for $200 \mathrm{~ms}$. Observers were instructed to indicate whether each presented stimulus was similar to a radial Glass pattern $\left(0^{\circ}\right.$ spiral angle) or a concentric Glass pattern $\left(90^{\circ}\right.$ spiral angle), by pressing one of two (left or right) buttons on a mouse. The buttons for different stimulus categories were counterbalanced across observers.

\section{fMRI sessions}

Observers participated in three scanning sessions: one high-signal stimulus session (i.e., observers were presented with stimuli at $80 \%$ signal level), one low-signal stimulus session (i.e., observers were presented with stimuli at $45 \%$ signal level) before training (after the psychophysical pretest), and one low-signal stimulus session after training (after the last psychophysical session). During scanning, observers performed the same categorization task as during the psychophysical sessions.

Each scanning session comprised eight experimental runs, each of which lasted $364 \mathrm{~s}$. Each run comprised eighteen 18-s-long stimulus blocks. A 10 s fixation block (i.e., only a fixation square was presented on the screen) was presented after every six stimulus blocks, as well as in the beginning and the end of each run. Each stimulus block was repeated three times in each run. The order of the blocks was randomized within each run, and each block was presented only once between two fixation blocks. Each stimulus block comprised 12 trials, including target and distractor stimuli. That is, 10 trials contained target stimuli presented at one of six conditions (i.e., spiral angles), whereas two trials contained distractor stimuli from another condition. Possible combinations of spiral angles (target/distractor stimuli) presented in a block were $10^{\circ} / 50^{\circ}$, $30^{\circ} / 60^{\circ}, 40^{\circ} / 80^{\circ}, 50^{\circ} / 10^{\circ}, 60^{\circ} / 30^{\circ}$, and $80^{\circ} / 40^{\circ}$. The presentation order of target and distractor stimuli within each block was randomized; one of the distractors was presented in the first six trials and the other in the last six trials. Each trial lasted $1.5 \mathrm{~s}$. Stimuli were presented for $200 \mathrm{~ms}$ each and separated by a $1300 \mathrm{~ms}$ interstimulus interval, during which observers made their response to the stimulus by pressing one of two keys. The color of the fixation square, which was presented during fixation blocks and throughout each trial, served as a cue for the motor response. If the cue was red, observers used the same key-category matching as during the psychophysical training sessions (e.g., left key for concentric pat- 

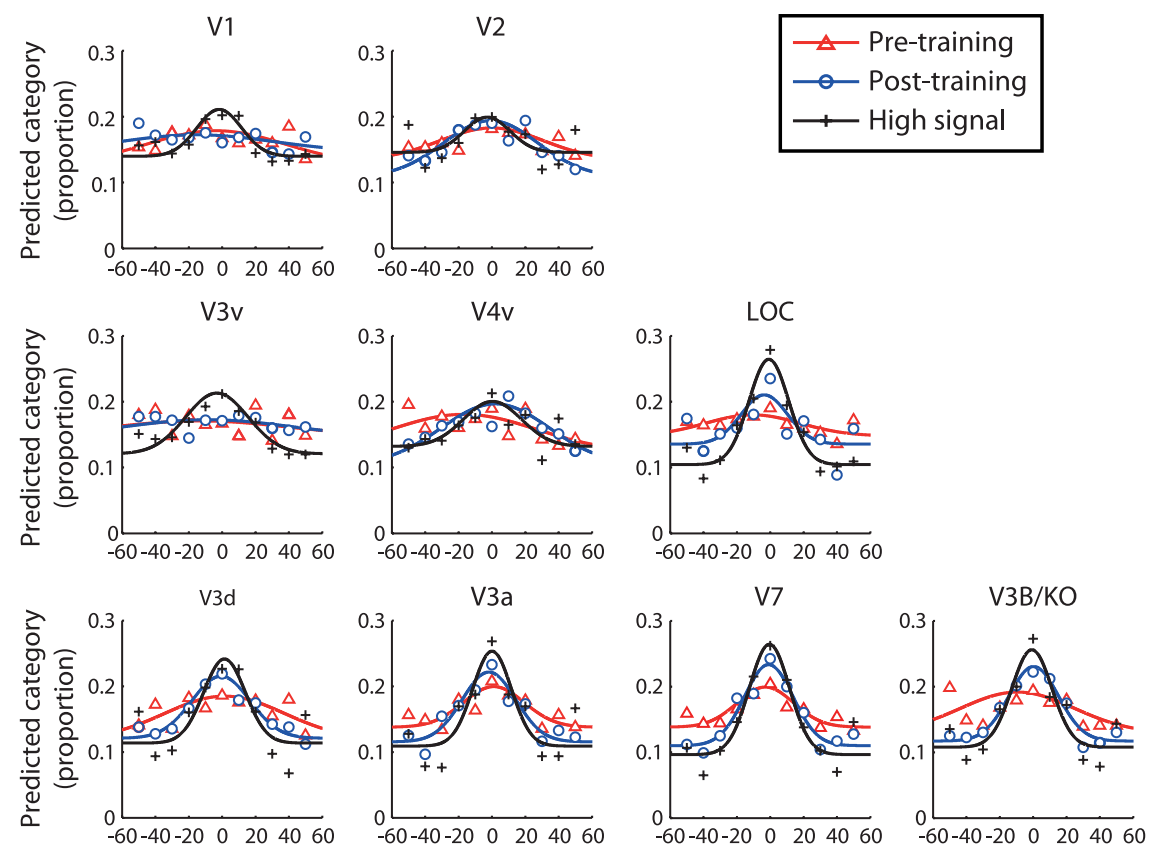

Spiral angle difference (degrees)

Figure 2. fMRI pattern-based tuning functions. The proportion of predictions made to each stimulus condition in terms of the difference in spiral angle between the viewed stimulus and the prediction. Symbols indicate average data across observers; solid lines indicate the best fit of a Gaussian to the data from 1000 bootstrap samples.

terns), whereas if the cue was green, observers switched finger-key matching (e.g., left key for radial patterns). The color of the fixation square changed after every six stimulus blocks (i.e., before each fixation block) and was counterbalanced across runs.

\section{fMRI data acquisition}

The experiments were conducted at the Birmingham University Imaging Centre using a 3 T Philips Achieva MRI scanner. T2*-weighted functional and T1-weighted anatomical $(1 \times 1 \times 1 \mathrm{~mm}$ resolution $)$ data were collected with an eight-channel head coil. Echo planar imaging data (gradient echo-pulse sequences) were acquired from 28 slices (repetition time, $2000 \mathrm{~ms}$; echo time, $34 \mathrm{~ms} ; 1.5 \times 1.5 \times 2 \mathrm{~mm}$ resolution). Slices were oriented near coronal covering the entire occipital and posterior temporal cortex.

\section{fMRI data analysis}

Data preprocessing. MRI data were processed using Brain Voyager QX (Brain Innovation B.V.). T1-weighted anatomical data were used for coregistration, three-dimensional cortex reconstruction, inflation, and flattening. Preprocessing of the functional data involved slice-scan time correction, three-dimensional head movement correction, temporal high-pass filtering (three cycles), and removal of linear trends. No spatial smoothing was performed on the functional data used for the multivariate analysis. The functional images were aligned to anatomical data under careful visual inspection, and the complete data were transformed into Talairach space (voxel size of $1 \times 1 \times 1 \mathrm{~mm}$, nearest-neighbor interpolation). Transforming the data into Talairach space ensured that the coordinates of the selected regions of interest (ROIs) for each individual subject were comparable with previous studies. When aligning the functional data to the anatomical scans, we used a nearest-neighbor interpolation method for resampling the data at high resolution $(1 \times 1 \times 1$ $\mathrm{mm}$ ) and included only the unique voxels for the pattern classification analysis. For each participant, the functional imaging data between sessions were coaligned, registering all volumes of each observer to the first functional volume. This procedure ensured a cautious registration across sessions.

Mapping regions of interest. For each individual observer, we identified retinotopic visual areas (V1, V2, V3d, V3a, V7, V3v, and V4v) based on standard mapping procedures (Engel et al., 1994; Sereno et al., 1995;
DeYoe et al., 1996). We also identified V3B/KO (kinetic occipital area) and the lateral occipital complex (LOC) in two independent scans. Area $\mathrm{V} 3 \mathrm{~B} / \mathrm{KO}$ was defined as the set of contiguous voxels anterior to $\mathrm{V} 3 \mathrm{a}$ that showed significantly stronger activation $(p<0.005)$ for kinetic boundaries than transparent motion (Dupont et al., 1997). LOC was defined as set of contiguous voxels in the ventral occipitotemporal cortex that showed significantly stronger activation $(p<0.005)$ for intact than scrambled images of objects (Kourtzi and Kanwisher, 2000).

\section{Multivoxel pattern analysis}

Voxel selection. For each observer and session, we selected voxels in each ROI (retinotopic areas, $\mathrm{V} 3 \mathrm{~B} / \mathrm{KO}$, and LOC) that showed stronger response to stimulus conditions than fixation $(p<0.05)$. To enable comparisons across ROIs and observers, we selected the average number of voxels across ROIs and observers with the highest difference between stimulus conditions $(p<0.05)$. This procedure resulted in the selection of 250 voxels per ROI, by which point classification accuracies had saturated in all ROIs. If an ROI had fewer than 250 voxels (4.94\% of cases across subjects and ROIs), we selected the classification accuracy at the maximum number of voxels in the region. The time course of each voxel was $z$-score normalized for each run and shifted by $4 \mathrm{~s}$ to account for the hemodynamic delay. For each pattern, we averaged the fMRI responses across all trials per block, resulting in 24 patterns per session for each condition and ROI.

Pattern classification. We trained linear support vector machine (SVM) classifiers using these patterns per ROI and calculated mean classification accuracies following a leave-one-run-out cross-validation procedure (supplemental Fig. S1A, available at www.jneurosci.org as supplemental material). That is, we trained binary classifiers on 21 training patterns and tested their accuracy on three test patterns per condition and ROI using an eightfold cross-validation procedure. For each crossvalidation, we selected voxels using only the training dataset, thus ensuring that the classifier was not confounded by using the same data for pattern classification and voxel selection. Also, to ensure that the classifier output did not simply result from univariate differences across conditions, we subtracted the grand mean response across voxels from each voxel.

To determine whether we could predict the viewed stimulus from the six possible stimulus conditions (i.e., spiral angles), we used multiple pairwise (one-against-one) binary classifiers (supplemental Fig. S1B, available at www.jneurosci.org as supplemental material) (Kamitani and Tong, 2005; Preston et al., 2008; Serences et al., 2009). In particular, we trained and tested all possible pairwise classifiers (15 comparisons) and collated their results for each test pattern. The predicted stimulus category corresponded to the category that received the fewest "votes against" when collating the results across all pairwise classifications. In the event of a tie, the prediction was randomly assigned to one of the categories. We expressed the accuracy of the six-way classifier as the proportion of test patterns for which it correctly predicted the viewed stimulus.

Pattern-based tuning functions. We examined the pattern of predictions made by the classifier when trained on a particular stimulus condition (i.e., spiral angle). We calculated the proportion of patterns for which the classifier predicted each stimulus condition from fMRI activity associated with each of the six different stimulus conditions. This gave us six sets of predictions for each of the six spiral angles: one prediction indicated the classification accuracy, whereas the rest indicated the classification errors. We plotted these 36 predictions as a function of the 
difference in spiral angle between the stimulus that evoked the fMRI response and the stimulus predicted by the classifier (supplemental Fig. $\mathrm{S} 1 \mathrm{~B}$, available at www.jneurosci.org as supplemental material). This allowed us to generate pattern-based tuning functions for spiral angle in each ROI. In particular, each proportion predicted $P(i)$ with stimulus distance (i.e., spiral angle difference) $i$ was calculated as follows:

$$
P(i)=\frac{n(i)}{N(i)}
$$

where $n(i)$ is the number of patterns predicted to have distance $i$ (from the stimulus condition), and $N(i)$ is the total number of patterns that is possible to be predicted to have distance $i$. We then fitted the averaged pattern-based tuning functions across observers using a Gaussian function:

$$
y=\frac{\alpha}{\sqrt{2 \pi s^{2}}} \exp \left(-\frac{(x-\mu)^{2}}{2 s^{2}}\right)+\beta,
$$

where $\alpha$ is the scaling parameter, $\mu$ is the mean, $s$ is the standard deviation, and $\beta$ is the baseline. Data for $\pm 70^{\circ}$ difference in spiral angle were excluded because they were derived from a single prediction $\left( \pm 10^{\circ}\right.$ vs $\pm 80^{\circ}$ ) resulting in outlier values.

Note that, for this approach, the area under each tuning function is not constrained to unity (or a constant number) because the proportion of patterns predicted is a function of the total number of patterns that is possible to be predicted for each condition (i.e., spiral angle difference) rather than the total number of patterns across conditions. To quantify the pattern-based tuning functions, we measured the amplitude (value at $x=0$ ) and the width (standard deviation $s$ ) of these functions based on 1000 bootstrap samples.

Voxel-based tuning functions. We generated tuning functions based on the fMRI response of individual voxels, as described previously (Serences et al., 2009). For each ROI and scanning session, we selected the same 250 voxels as for the pattern-based tuning functions following the same procedure for voxel selection and using only the training data. The time course of each voxel was $z$-score normalized for each run and shifted by $4 \mathrm{~s}$ to account for the hemodynamic delay. We then determined the preference of each voxel by the stimulus condition that evoked the largest mean response when considering data from all experimental runs except one (test run). Then using the data from the test run, we determined the response of each voxel in each condition by the difference (in spiral angle) between the stimulus condition and the preference of the voxel. Akin to the MVPA procedure, we averaged the results from this leaveone-run-out eightfold cross-validation procedure to obtain voxel-based tuning functions for each observer and ROI. We then fitted the average tuning functions across observers with the Gaussian function and estimated the amplitude and the width of these functions from 1000 bootstrap samples.

\section{Results}

\section{Behavioral results}

We tested the observers' ability to categorize global form patterns as radial or concentric when stimuli were presented at high versus low signal before and after training (Fig. $1 A$ ). Our results showed that training improved the observers' sensitivity in discriminating visual forms, that is, the $78 \%$ threshold performance for low-signal stimuli was reduced after training (Fig. $1 B$ ). A repeated-measures ANOVA showed higher standard deviation (estimated from cumulative Gaussian fits on individual subject data) for low- than high-signal stimuli before training $\left(F_{(1,7)}=14.35, p<0.01\right)$, which decreased significantly after training $\left(F_{(1,7)}=10.24, p<\right.$ $0.05)$. Similar results were observed during testing in the laboratory (supplemental Fig. S2, available at www.jneurosci.org as supplemental material), indicating that training enhanced the observers' sensitivity to stimulus category.
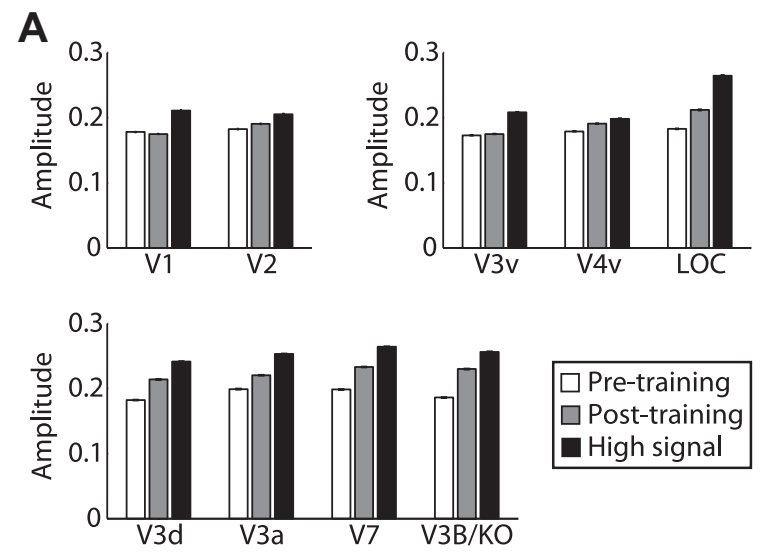

B
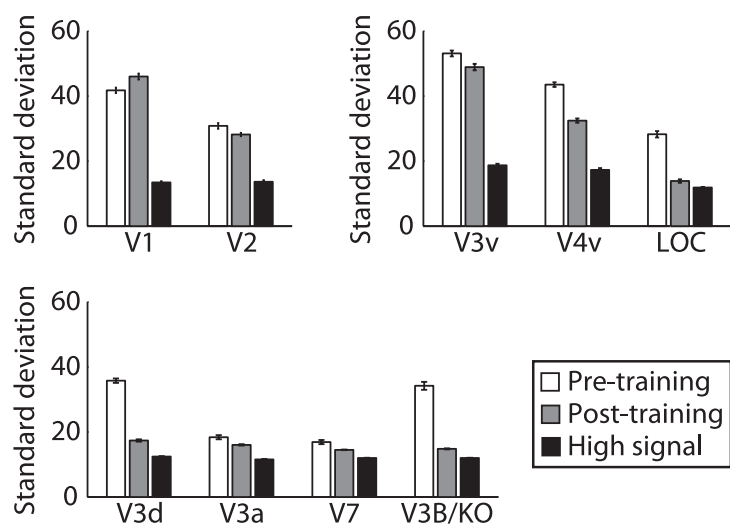

Figure 3. Measures of fMRI pattern tuning. $\boldsymbol{A}, \boldsymbol{B}$, Amplitude $(\boldsymbol{A})$ and width $(\boldsymbol{B})$ (standard deviation of the Gaussian fit) of the pattern-based tuning functions. Error bars indicate $95 \%$ confidence intervals calculated from 1000 bootstrap samples.

\section{fMRI results: pattern-based tuning functions}

We used multivoxel pattern classification (Haynes and Rees, 2005; Kamitani and Tong, 2005) to investigate which visual areas encode selective information about shape category (concentric, radial Glass patterns) as determined by the orientation of the local stimulus dipoles (i.e., spiral angle). In particular, we used a six-way linear SVM classifier to discriminate fMRI responses evoked by each stimulus condition from fMRI responses for the other five conditions. We calculated the predictions of the classifier for each stimulus condition using the fMRI activity associated with each of the six different stimulus conditions. From these predictions, we generated pattern-based tuning functions across spiral angle in each ROI (Fig. 2). For high-signal stimuli, we observed a tuned response across visual areas. That is, the classifiers mispredicted stimuli at similar spiral angles more frequently than stimuli at dissimilar spiral angles, suggesting fMRI selective responses for visual forms differing in local orientation signals. To assess the effect of learning on fMRI selectivity for visual forms, we compared the amplitude and the width (i.e., standard deviation of the Gaussian fits) of the pattern-based tuning functions for high-signal stimuli with those for low-signal stimuli before and after training. The amplitude at $x=0$ indicates accuracy for each predicted stimulus category relating to the classification accuracy of the six-way MVPA (supplemental Fig. S3, available at www.jneurosci.org as supplemental material).

Consistent with the behavioral results, we observed higher amplitude for high- than low-signal stimuli before training in higher visual areas. However, after training, the amplitude for low-signal stimuli increased significantly in higher dorsal and 

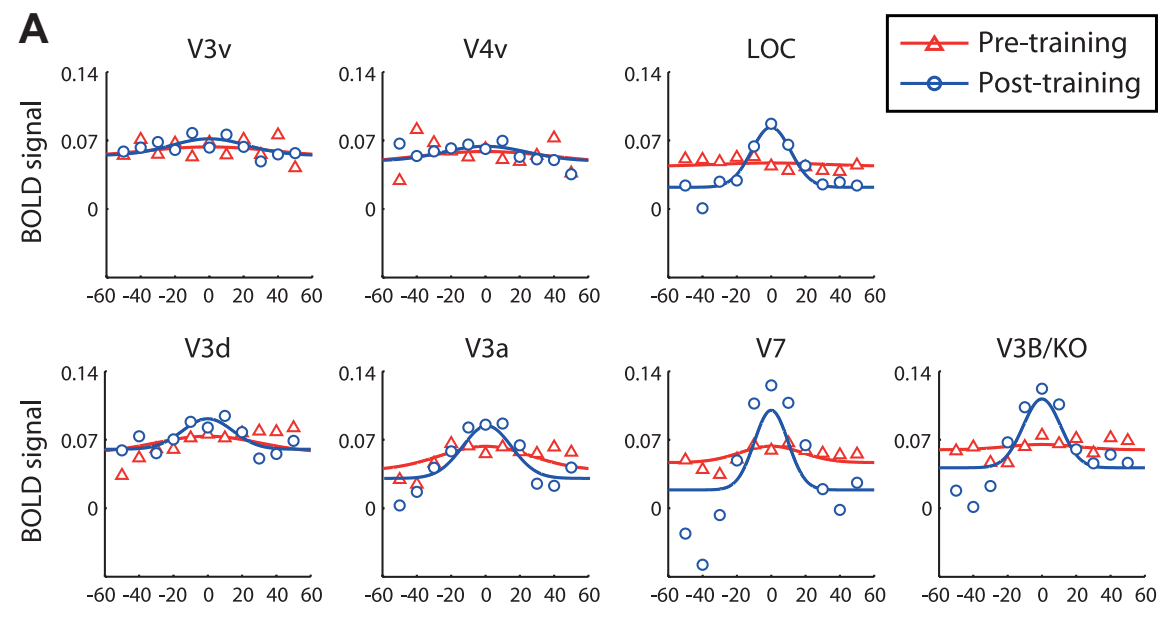

Spiral angle difference (degrees)
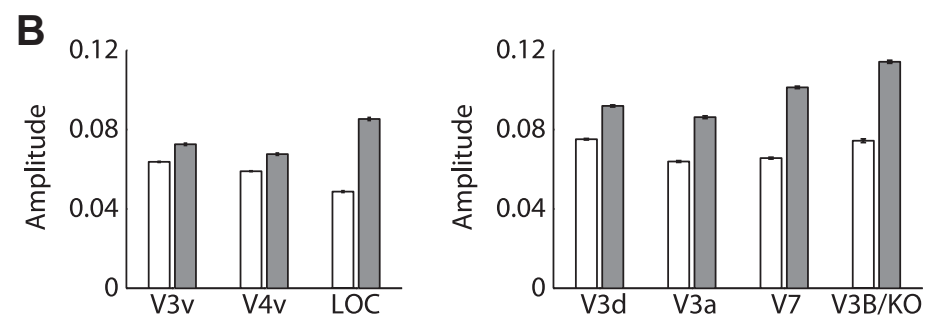

\section{C}
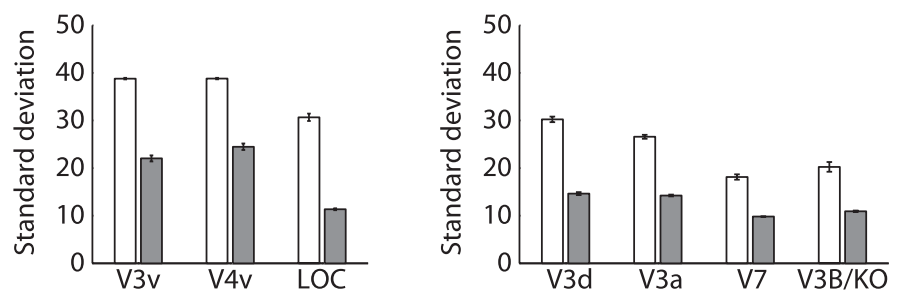

Figure 4. Voxel-based tuning functions. $\boldsymbol{A}, \mathrm{BOLD}$ signal (average across voxels) plotted against the difference in spiral angle between each stimulus condition and the preferred condition of each voxel. $\boldsymbol{B}, \boldsymbol{C}$, Amplitude $(\boldsymbol{B})$ and width $(\boldsymbol{C})$ (standard deviation of the Gaussian fit) of the voxel-based tuning functions. Error bars indicate $95 \%$ confidence intervals calculated from 1000 bootstrap samples.

ventral areas (Fig. 3A). A repeated-measures ANOVA showed significant differences across sessions in dorsal $\left(F_{(2,14)}=15.33\right.$, $p<0.001)$ and ventral $\left(F_{(2,14)}=27.26, p<0.001\right)$ but not early $(\mathrm{V} 1, \mathrm{~V} 2)$ visual areas $\left(F_{(2,14)}=2.02, p=0.17\right)$. For dorsal visual areas, amplitude was higher for high- than low-signal stimuli before training $\left(F_{(1,7)}=64.49, p<0.001\right)$, but it increased significantly for low-signal stimuli after training $\left(F_{(1,7)}=7.84, p<\right.$ $0.05)$. Similar results were observed in the LOC rather than earlier ventral areas $(\mathrm{V} 3 \mathrm{v}, \mathrm{V} 4)$, as indicated by a significant interaction between session and ventral regions $\left(F_{(4,28)}=3.13, p<0.05\right)$.

Next, we observed narrower tuning of the pattern-based functions for high- than low-signal stimuli before training in higher visual areas (Fig. 3B). However, training enhanced the tuning width in both dorsal and ventral areas. A repeated-measures ANOVA showed significant differences in the tuning width across sessions for dorsal $\left(F_{(2,14)}=20.09, p<0.001\right)$ and ventral $\left(F_{(2,14)}=16.90, p<0.001\right)$ areas but not early $(\mathrm{V} 1, \mathrm{~V} 2)$ visual areas $\left(F_{(2,14)}=1.15, p=0.27\right)$. For both dorsal $\left(F_{(1,7)}=64.05\right.$, $p<0.0 .001)$ and ventral $\left(F_{(1,7)}=31.99, p<0.01\right)$ areas, the tuning width was narrower for high- than low-signal stimuli before training. However, after training, the tuning width for lowsignal stimuli decreased significantly in dorsal $\left(F_{(1,7)}=6.02, p<\right.$ $0.05)$ and ventral $\left(F_{(1,7)}=12.27, p<0.01\right)$ areas.
In summary, our results show that training results in behavioral improvement and changes in the tuning of multivoxel patterns in higher dorsal and ventral visual areas. It is interesting to note that, although behavioral and fMRI sensitivity for low-signal stimuli improved with training, it did not reach the levels of sensitivity for high-signal stimuli. In particular, the $78 \%$ threshold was lower for high- than low-signal stimuli after training $\left(F_{(1,7)}=19.44, p<0.01\right)$, suggesting that observers' sensitivity for discriminating global forms was higher for high- than low-signal stimuli. Similarly, the amplitude of the pattern-based tuning functions was higher $\left(F_{(1,7)}=\right.$ $10.87, p<0.05)$ whereas the width significantly lower $\left(F_{(1,7)}=6.78, p<0.05\right)$ for high- than low-signal stimuli after training. It is possible that more extensive training would result in equivalent performance and fMRI pattern-based tuning for high- and low-signal stimuli.

\section{Control analyses}

To discern learning-dependent changes to fMRI signals related to preferred compared with nonpreferred shape categories, we compared the amplitude and width of pattern-based tuning functions. However, these parameters can be coupled; that is, changes in tuning width can relate to changes in amplitude. Therefore, we performed two additional analyses. First, we examined the pattern of mispredictions by excluding prediction data from the preferred shape category (i.e., zero difference in spiral angle). Regression analysis (supplemental Fig. S3, available at www.jneurosci.org as supplemental material) showed steeper slopes (i.e., reduced mispredictions far from the preferred category) across higher dorsal and ventral areas after training, indicating narrower tuning independent of changes in the peak of the pattern-based tuning functions. Second, we tested learningdependent changes in the preferences of individual voxels for shape categories (Fig. 4). This method (Serences et al., 2009) does not rely on probability distributions and produces voxel-based tuning functions for which amplitude and standard deviation are independent. In agreement with the MVPA results, we observed changes in both the amplitude and standard deviation of tuning in higher dorsal and ventral areas. These findings provide additional evidence for learning-dependent changes not only in the overall responsiveness of large neural populations (at the level of single voxels or patterns) to trained stimuli but also in the neural sensitivity to shape categories.

To avoid confounds and ensure that our data treatment was appropriate, we conducted the following additional analyses. First, we tested for differences in the overall fMRI responsiveness that could result from differences in task difficulty across scanning sessions. Analysis of the percentage signal change from fixation baseline across cortical regions did not show any significant differences across scanning sessions $\left(F_{(2,14)}=1.08, p=0.37\right)$, 
suggesting that differences in the pattern-based tuning functions could not be attributed to differences in the overall fMRI signal. Second, to ensure that our classification approach was not overpowered and did not suffer from any bias, we ran the classification with the data labels shuffled. The results for the classification of 1000 permutation of the six-way classifier (supplemental Table $\mathrm{S} 1$, available at www.jneurosci.org as supplemental material) were at chance. Finally, analysis of eye movement data collected during scanning did not show any significant differences between sessions in the eye position or number of saccades (supplemental Fig. S5, available at www.jneurosci.org as supplemental material), suggesting that differences in the pattern-based tuning functions across sessions could not be significantly attributed to eye movement differences.

\section{Discussion}

Our findings demonstrate that training alters the observers' sensitivity to visual forms and fMRI selectivity in higher dorsal and ventral visual areas. In particular, we show that training on lowsignal stimuli increases the amplitude but reduces the width of pattern-based tuning in higher dorsal and ventral visual areas. Increased amplitude after training indicates higher stimulus discriminability that may relate to enhanced neural responses for the preferred stimulus category at the level of large neural populations across voxels. Reduced tuning width after training indicates fewer classification mispredictions, suggesting that learning decreases neural responses to nonpreferred stimuli. Thus, our findings suggest that learning of visual patterns is implemented in the human visual cortex by enhancing the response to the preferred stimulus category, whereas reducing the response to nonpreferred stimuli.

Our findings advance our understanding of learning brain mechanisms in two main respects. First, we provide evidence for learning-dependent changes related to neural sensitivity rather than simply overall responsiveness (i.e., increased or decreased fMRI responses) to trained stimuli as reported in previous imaging studies (Kourtzi et al., 2005; Sigman et al., 2005; Op de Beeck et al., 2006; Mukai et al., 2007; Yotsumoto et al., 2008). This previous work does not allow us to discern whether learningdependent changes in fMRI signals relate to changes in the overall magnitude of neural responses or changes in neuronal selectivity of neural populations. Previous work using fMRI adaptation has suggested selectivity changes related to learning (Jiang et al., 2007; Gillebert et al., 2009). Here, we take advantage of the sensitivity of high-resolution fMRI recordings and MVPA methods to discern the mechanisms that mediate learning-dependent changes in visual selectivity. This combination of methods allows us to discern whether learning changes the magnitude of responses to preferred or nonpreferred stimuli by comparing fMRI tuning functions before and after training. Although the low spatial resolution of fMRI compared with neurophysiology does not allow us to investigate learning-dependent changes at the level of single neurons, our methodology provides sensitive tools for testing how learning shapes the fine-tuned representation of visual forms across large neural populations.

Second, our findings provide novel evidence for the role of dorsal areas in learning visual forms. Although the evidence for experience-dependent plasticity in V1 remains controversial (Crist et al., 2001; Schoups et al., 2001; Ghose et al., 2002; Furmanski et al., 2004; Li et al., 2004), recent work indicates that learning shapes visual processing in ventral stream areas. In particular, training is shown to result in greater changes in orientation tuning in V4 than in V1 (Yang and Maunsell, 2004; Raiguel et al., 2006). However, our results demonstrate learningdependent changes in fMRI selectivity in dorsal visual areas, consistent with our previous work showing that these areas are involved in the integration of local orientation signals into global forms (Ostwald et al., 2008). Furthermore, our results show learning-dependent changes in fMRI selectivity in the LOC, consistent with the role of learning in shaping inferotemporal processing of complex visual features (Sigala and Logothetis, 2002; Freedman et al., 2006), multiple-part configurations (Baker et al., 2002), and objects (Logothetis et al., 1995; Rolls, 1995; Kobatake et al., 1998).

Thus, our findings suggest that learning alters the tuning of activation patterns in regions selective for task-relevant visual features. We have shown previously that the categorization of visual forms is achieved by integrating local visual features and configurations in dorsal visual areas, whereas global form structure in the LOC (Ostwald et al., 2008). Here we propose that learning shapes these processes by decreasing responses to distractor stimuli during the integration of visual forms, whereas enhancing responses for the selective representation of behaviorally relevant stimuli. Recent neurophysiology work suggests that these learning-dependent changes may be mediated by changes in the readout of signals rather than stimulus encoding in visual areas (Law and Gold, 2008). Although, the high-resolution fMRI used in our study limited our recordings to the visual cortex, it is possible that higher frontoparietal circuits may modulate neural plasticity and optimize visual processing through attention-gated learning mechanisms (Hochstein and Ahissar, 2002; Roelfsema and van Ooyen, 2005). Additional work using multimodal imaging (e.g., EEG-fMRI measurements) is necessary for understanding these interactions across cortical circuits and the spatiotemporal dynamics that mediate learning-dependent plasticity in the human brain.

\section{References}

Baker CI, Behrmann M, Olson CR (2002) Impact of learning on representation of parts and wholes in monkey inferotemporal cortex. Nat Neurosci 5:1210-1216.

Crist RE, Li W, Gilbert CD (2001) Learning to see: experience and attention in primary visual cortex. Nat Neurosci 4:519-525.

DeYoe EA, Carman GJ, Bandettini P, Glickman S, Wieser J, Cox R, Miller D, Neitz J (1996) Mapping striate and extrastriate visual areas in human cerebral cortex. Proc Natl Acad Sci U S A 93:2382-2386.

Dupont P, De Bruyn B, Vandenberghe R, Rosier AM, Michiels J, Marchal G, Mortelmans L, Orban GA (1997) The kinetic occipital region in human visual cortex. Cereb Cortex 7:283-292.

Engel SA, Rumelhart DE, Wandell BA, Lee AT, Glover GH, Chichilnisky EJ, Shadlen MN (1994) fMRI of human visual cortex. Nature 369:525.

Freedman DJ, Riesenhuber M, Poggio T, Miller EK (2006) Experiencedependent sharpening of visual shape selectivity in inferior temporal cortex. Cereb Cortex 16:1631-1644.

Furmanski CS, Schluppeck D, Engel SA (2004) Learning strengthens the response of primary visual cortex to simple patterns. Curr Biol 14: 573-578.

Ghose GM, Yang T, Maunsell JH (2002) Physiological correlates of perceptual learning in monkey V1 and V2. J Neurophysiol 87:1867-1888.

Gilbert CD, Sigman M, Crist RE (2001) The neural basis of perceptual learning. Neuron 31:681-697.

Gillebert CR, Op de Beeck HP, Panis S, Wagemans J (2009) Subordinate categorization enhances the neural selectivity in human object-selective cortex for fine shape differences. J Cogn Neurosci 21:1054-1064.

Glass L (1969) Moire effect from random dots. Nature 223:578-580.

Haynes JD, Rees G (2005) Predicting the orientation of invisible stimuli from activity in human primary visual cortex. Nat Neurosci 8:686-691.

Hochstein S, Ahissar M (2002) View from the top: hierarchies and reverse hierarchies in the visual system. Neuron 36:791-804.

Jiang X, Bradley E, Rini RA, Zeffiro T, Vanmeter J, Riesenhuber M (2007) 
Categorization training results in shape- and category-selective human neural plasticity. Neuron 53:891-903.

Kamitani Y, Tong F (2005) Decoding the visual and subjective contents of the human brain. Nat Neurosci 8:679-685.

Kobatake E, Wang G, Tanaka K (1998) Effects of shape-discrimination training on the selectivity of inferotemporal cells in adult monkeys. J Neurophysiol 80:324-330.

Kourtzi Z, DiCarlo JJ (2006) Learning and neural plasticity in visual object recognition. Curr Opin Neurobiol 16:152-158.

Kourtzi Z, Kanwisher N (2000) Cortical regions involved in perceiving object shape. J Neurosci 20:3310-3318.

Kourtzi Z, Betts LR, Sarkheil P, Welchman AE (2005) Distributed neural plasticity for shape learning in the human visual cortex. PLoS Biol 3:e204.

Law CT, Gold JI (2008) Neural correlates of perceptual learning in a sensory-motor, but not a sensory, cortical area. Nat Neurosci 11:505-513.

Li S, Mayhew SD, Kourtzi Z (2009) Learning shapes the representation of behavioral choice in the human brain. Neuron 62:441-452.

Li W, Piëch V, Gilbert CD (2004) Perceptual learning and top-down influences in primary visual cortex. Nat Neurosci 7:651-657.

Logothetis NK, Pauls J, Poggio T (1995) Shape representation in the inferior temporal cortex of monkeys. Curr Biol 5:552-563.

Mukai I, Kim D, Fukunaga M, Japee S, Marrett S, Ungerleider LG (2007) Activations in visual and attention-related areas predict and correlate with the degree of perceptual learning. J Neurosci 27:11401-11411.

Op de Beeck HP, Baker CI, DiCarlo JJ, Kanwisher NG (2006) Discrimination training alters object representations in human extrastriate cortex. J Neurosci 26:13025-13036.

Ostwald D, Lam JM, Li S, Kourtzi Z (2008) Neural coding of global form in the human visual cortex. J Neurophysiol 99:2456-2469.
Preston TJ, Li S, Kourtzi Z, Welchman AE (2008) Multivoxel pattern selectivity for perceptually relevant binocular disparities in the human brain. J Neurosci 28:11315-11327.

Raiguel S, Vogels R, Mysore SG, Orban GA (2006) Learning to see the difference specifically alters the most informative V4 neurons. J Neurosci 26:6589-6602.

Roelfsema PR, van Ooyen A (2005) Attention-gated reinforcement learning of internal representations for classification. Neural Comput 17:2176-2214.

Rolls ET (1995) Learning mechanisms in the temporal lobe visual cortex. Behav Brain Res 66:177-185.

Schoups A, Vogels R, Qian N, Orban G (2001) Practising orientation identification improves orientation coding in V1 neurons. Nature 412: $549-553$.

Serences JT, Saproo S, Scolari M, Ho T, Muftuler LT (2009) Estimating the influence of attention on population codes in human visual cortex using voxel-based tuning functions. Neuroimage 44:223-231.

Sereno MI, Dale AM, Reppas JB, Kwong KK, Belliveau JW, Brady TJ, Rosen BR, Tootell RB (1995) Borders of multiple visual areas in humans revealed by functional magnetic resonance imaging. Science 268:889-893.

Sigala N, Logothetis NK (2002) Visual categorization shapes feature selectivity in the primate temporal cortex. Nature 415:318-320.

Sigman M, Pan H, Yang Y, Stern E, Silbersweig D, Gilbert CD (2005) Topdown reorganization of activity in the visual pathway after learning a shape identification task. Neuron 46:823-835.

Yang T, Maunsell JH (2004) The effect of perceptual learning on neuronal responses in monkey visual area V4. J Neurosci 24:1617-1626.

Yotsumoto Y, Watanabe T, Sasaki Y (2008) Different dynamics of performance and brain activation in the time course of perceptual learning. Neuron 57:827-833. 